\title{
Analysis of ROR1 Protein Expression in Mice with Reconstituted Human Immune System Components
}

\author{
Carol S. Leung (iD ${ }^{1,2}$ \\ ${ }^{1}$ Department of Hematology, University College London Cancer Institute, University College London, London, UK \\ ${ }^{2}$ Ludwig Institute for Cancer Research, Nuffield Department of Medicine, University of Oxford, Oxford, UK \\ Correspondence should be addressed to Carol S. Leung; carol.leung@ludwig.ox.ac.uk
}

Received 15 September 2017; Revised 1 February 2018; Accepted 11 March 2018; Published 18 April 2018

Academic Editor: Kurt Blaser

Copyright (c) 2018 Carol S. Leung. This is an open access article distributed under the Creative Commons Attribution License, which permits unrestricted use, distribution, and reproduction in any medium, provided the original work is properly cited.

\begin{abstract}
Receptor tyrosine kinase-like orphan receptor 1 (ROR1) is an oncofetal antigen expressed on multiple tumors and has no significant expression on normal human tissues. ROR1 is highly upregulated in chronic lymphocytic leukemia (CLL) B cells. NOD-scid IL2 $\mathrm{rg}^{-1-}$ (NSG) mice engrafted with human CD34 $4^{+}$hematopoietic progenitor cells (huNSG) achieved multilineage human immune cell reconstitution including B cells, T cells, NK cells, and DCs. Like the CLL patients, huNSG mice have abnormally high percentage of CD5-expressing B cells in the periphery. In light of this, we aim to determine whether ROR1 is expressed on huNSG B cells. Using flow cytometry analysis, we found that ROR1 was highly expressed in a proportion of bone marrow, spleen, and blood B cells, which were mostly immature B cells. Transplantation of the oncogene TCL-1-transduced $\mathrm{CD}_{4} 4^{+}$cells in neonatal NSG mice did not increase the frequency of ROR1-expressing B cells, but the mouse with the highest engraftment of transduced cells developed a tumor-like lump consisting of a high percentage of ROR1-expressing B cells. This study highlights the potential use of huNSG mice to study B cell malignant diseases and to evaluate immunotherapeutics targeting ROR1.
\end{abstract}

\section{Introduction}

Receptor tyrosine kinase-like orphan receptor 1 (ROR1) is an oncofetal antigen expressed in a number of malignancies. The overexpression of ROR1 in malignancy was first identified on chronic lymphocytic leukemia (CLL) B cells [1] and was subsequently found in many other hematological malignancies [2-4] and solid tumors [5]. It has been shown that ROR1 could play a crucial role in tumorigenesis [6] and cell migration [7]. As ROR1 has expression on tumor cells but not on normal human tissues except at low levels in adipose tissues, parathyroid, pancreatic islet cells, and some regions of the gastrointestinal tract [8], this makes it an attractive antigen target for cancer therapy. Indeed, a number of ROR1-specific monoclonal antibodies and chimeric antigen receptor (CAR) $\mathrm{T}$ cells have been developed and are under testing $[9,10]$. However, a preclinical small animal model is currently lacking to evaluate ROR1-targeted immunotherapies.

Immunodeficient NOD-scid IL2 $\mathrm{rg}^{-/-}$(NSG) mice engrafted with human fetal liver-derived $\mathrm{CD} 34^{+}$hematopoietic progenitor cells (huNSG) achieved multilineage human immune cell reconstitution including B cells, T cells, natural killer (NK) cells, and dendritic cells (DCs) [11]. These so called humanized mice are a powerful tool to study human infectious diseases, hematopoiesis, and model immune system tumor interaction and can be used to evaluate novel antitumor immunotherapies $[12,13]$. However, incomplete B cell development in huNSG mice has been documented [14]. Like CLL patients, huNSG mice have abnormally high frequency of $\mathrm{B}$ cells in the periphery, and a subset of B cells expresses CD5. In light of these, we hypothesized that huNSG mice have a high proportion of $\mathrm{ROR} 1^{+} \mathrm{B}$ cells and could represent a ROR $1^{+}$tumor model in vivo.

Here, we evaluated ROR1 protein expression in engrafted human immune cells in 3 different cohorts of huNSG mice. We analyzed the phenotypes and characteristics of ROR1expressing B cells. Moreover, CD $34^{+}$human hematopoietic progenitor cells transduced with the oncogene $\mathrm{T}$ cell leukemia/lymphoma 1 (TCL-1) were transplanted in neonatal NSG mice to study the effect of this oncogene on inducing ROR1-expressing tumors in huNSG mice. 


\section{Materials and Methods}

2.1. Generation of huNSG Mice. NOD, Cg-Prkdscid $I l 2 \mathrm{rg}^{t m 1 \mathrm{Wjl}} / \mathrm{SzJ}$ (NSG), mice were obtained from The Jackson Laboratory and raised under specific pathogen-free conditions. Human fetal liver samples were obtained from Advanced Bioscience Resources; human $\mathrm{CD} 34^{+}$hematopoietic progenitor cells were isolated using the human CD34 MicroBead kit (Miltenyi Biotec). HuNSG mice were generated as previously described [11]. Animal protocols were approved by the UK Home Office, and all animal experiments were performed in accordance with institutional guidelines under protocol number 70/7295. This study was also approved by the Research Ethics Committee with REC reference number 16/EE/0043.

2.2. Lentiviral Vector Construction and Human $\mathrm{CD} 34^{+}$Cell Transduction. The coding sequence of the human TCL-1 gene was synthesized by Genscript and cloned into the lentiviral vector pCCL-GFP (Addgene) with the EF1 $\alpha$ promoter. This created pCCL-EF1 $\alpha$-TCL1-GFP. Lentivirus expressing TCL-1 was then prepared by cotransfecting 293T cells with pCCL-EF1 $\alpha$-TCL1-GFP, lentiviral packaging and envelope plasmids. Lentivirus-containing supernatant was harvested after 48 hours and was used to transduce human $\mathrm{CD} 34^{+}$cells. Frozen CD $34^{+}$cells were thawed and cultured in StemPro-34 SFM complete medium (Gibco) with the cytokines SCF and IL-3, in combination with the lentiviral vectors. After 24 hours of transduction, culture media were changed and cells were cultured for another 72 hours. GFP expression was evaluated by flow cytometry before injecting these cells into NSG mice.

2.3. Flow Cytometry. Cells were washed in PBS and stained for 20 minutes with conjugated antibodies or isotype control obtained from BioLegend, eBioscience, or BD. The following purified mouse anti-human antibodies were used for staining: anti-CD45-Pacific Blue, anti-CD45-BV605, antiCD19-PE-Cy7, anti-CD19-APC-Cy7, CD10-BV605, antiCD27-PE-Cy7, anti-IgD-PE, anti-ROR1-APC (clone 2A2), anti-CD5-PerCP-Cy5.5, anti-CD38-BV711, anti-CD23FITC, anti-NKp46-FITC, anti-CD3-Pacific Blue, anti-CD4APC-Cy7, and anti-CD8-PE. Live and dead cell staining was performed with aqua fluorescent reactive dye from Invitrogen. Peripheral blood mononuclear cells (PBMCs) from heathy donors and CLL patients were kindly provided by Dr. Vania Coelho, University College London. Flow cytometry analysis was done using a BD LSRII Fortessa using FACSDiva software (BD Biosciences), and data were analyzed with FlowJo (Tree Star).

2.4. B Cell Proliferation Assay. Frozen splenocytes from huNSG mice in the same reconstitution cohort were thawed and stained with $5 \mu \mathrm{M}$ CellTrace Violet (Invitrogen) and washed, and 200,000 live splenocytes were plated in $200 \mu \mathrm{l}$ of culture medium in each well of 96-well flat-bottom plates. The cells were stimulated with $5 \mu \mathrm{g} / \mathrm{ml} \mathrm{CpG} \mathrm{ODN} 2006$ (InvivoGen), $167 \mathrm{ng} / \mathrm{ml}$ pokeweed mitogen (PWM) extract (Sigma), and 1/2400 fixed Staphylococcus aureus cells (SAC) (Calbiochem) for 96 hours and analyzed by flow cytometry.
2.5. Western Blot. Untransduced or transduced $\mathrm{CD} 34^{+}$ hematopoietic progenitor cells by lentivirus expressing TCL-1 were lysed by RIPA buffer containing protease inhibitor (Sigma). Protein extracts were separated by Bis-Tris gels and transferred to the PVDF membrane by Western blotting and probed with TCL-1-specific monoclonal antibody clone 1-21 (Cell Signaling). Goat anti-mouse IgG coupled with HRP was used as a secondary antibody. Blots were developed using the ECL kit (GE Healthcare), and protein bands were detected on X-ray film.

\section{Results}

3.1. ROR1 Expression on B Cells in huNSG Mice. We first examined the ROR1 surface expression on reconstituted human immune cells in huNSG mice. These mice were generated by engrafting newborn immunodeficient NSG mice with human fetal liver-derived $\mathrm{CD} 34^{+}$hematopoietic progenitor cells $[11,15]$. We generated 3 cohorts of huNSG mice with human $\mathrm{CD} 34^{+}$hematopoietic progenitor cells derived from 3 different fetal liver tissues. Most of the huNSG mice achieved a frequency of more than $50 \%$ of human $\mathrm{CD} 45^{+}$cells in total leukocytes after 3 months of reconstitution, with engraftment of $\mathrm{CD} 19^{+} \mathrm{B}$ cells, $\mathrm{CD}^{+} \mathrm{T}$ cells, and $\mathrm{NKp} 6^{+}$NK cells (Figure 1). Afterwards, we investigated the ROR1 surface expression on engrafted human immune cells in huNSG mice, comparing such expression with that in a human healthy donor and a CLL patient. PBMCs from the healthy donor did not express ROR1 while a high proportion of ROR1-expressing B cells was observed in the PBMCs of the CLL patient (Figure 2(a)). Interestingly, we found a high percentage of $\mathrm{CD} 19^{+} \mathrm{ROR}^{+} \mathrm{B}$ cells in huNSG mice, especially in the bone marrow and spleen. This was observed in mice from all 3 cohorts, with a mean of $47.2 \%$ in the bone marrow, $13.7 \%$ in the spleen, and $2.0 \%$ in the blood (Figure 2(b)). On the other hand, only a negligible amount of $\mathrm{CD} 45^{+} \mathrm{CD} 19^{-}$immune cells expressed ROR1.

3.2. Frequency of ROR1-Expressing B Cells Maintained in huNSG Mice. The abnormally high percentage of ROR1 ${ }^{+}$ $\mathrm{B}$ cells may have been caused by the incomplete $\mathrm{B}$ cell development in huNSG mice [14]. A previous study has suggested that human B cell maturity improves with time following the reconstitution [16], so we tested if the frequency of ROR $1^{+} \mathrm{B}$ cells changes over time after the transplantation of human cells. In Figure 3(a), the percentage of $\mathrm{ROR}^{+} \mathrm{CD} 19^{+} \mathrm{B}$ cells in the periphery remained largely unchanged between 3 and 8 months after human progenitor cell engraftment. This was observed in both individual cohorts and the pooled cohort. In addition, the frequency of this subset was also stable over time in the bone marrow and spleen of huNSG mice, although it should be noted that each cohort only contributed to one single time point (Figure 3(b)). Also, the reconstituted frequency of $\mathrm{ROR} 1^{+} \mathrm{CD} 19^{+} \mathrm{B}$ cells correlated positively with CD19 reconstitution $(r=0.53)$ and negatively with CD3 reconstitution $(r=-0.74)$, but to a lesser extent with NKp $46^{+}$NK cell reconstitution $(r=-0.31)$. 


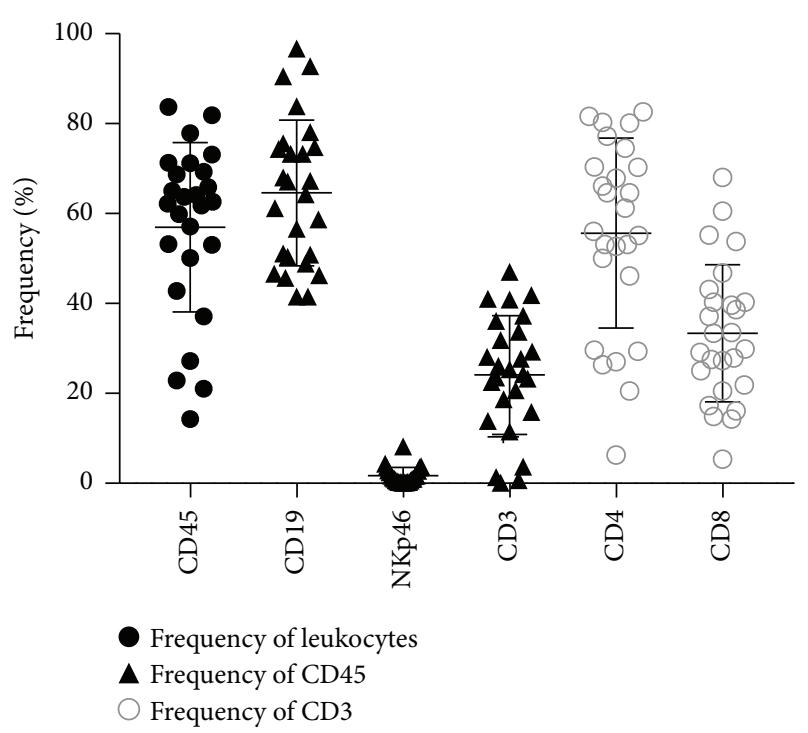

Figure 1: NOD-scid IL2 $\mathrm{rg}^{-/-}$(NSG) mice injected with fetal liverderived $\mathrm{CD}_{3} 4^{+}$hematopoietic progenitor cells were reconstituted with human immune cells. Peripheral blood of reconstituted NSG mice was analyzed 3 months after injection of human hematopoietic progenitor cells. The frequencies of different immune cell compartments are indicated. Frequencies of human $\mathrm{CD} 45^{+}$cells within the leukocyte gate, frequencies of $\mathrm{CD} 19^{+} \mathrm{B}$ cells, NKp $46^{+} \mathrm{NK}$ cells, and $\mathrm{CD}^{+}{ }^{+} \mathrm{T}$ cells within human $\mathrm{CD} 45^{+}$ cells, and frequencies of $\mathrm{CD}^{+}$and $\mathrm{CD}^{+} \mathrm{T}$ cells within $\mathrm{CD}^{+}$cells are shown. Horizontal lines represent the mean and SD. Data are from 3 different reconstitution cohorts with $\mathrm{CD}_{3} 4^{+}$cells derived from 3 different fetal liver tissues.

3.3. ROR1-Expressing B Cells Were Mostly Immature B Cells. We examined the phenotype of the ROR $1^{+} \mathrm{CD} 19^{+} \mathrm{B}$ cells in huNSG mice. In the bone marrow, ROR1-expressing B cells were mainly $\mathrm{CD} 27$ and $\mathrm{IgD}$ double negative (99.2\%), $\mathrm{CD} 10^{+}$, and $\mathrm{CD} 38^{+}$but were $\mathrm{CD}^{-}$(Figure $4(\mathrm{a})$ ). The majority of the splenic ROR1-expressing $\mathrm{B}$ cells were also $\mathrm{CD} 27^{-} \mathrm{IgD}^{-}$and $\mathrm{CD}_{10} 0^{+} \mathrm{CD} 38^{+}$, with slightly larger $\mathrm{CD} 27^{-} \mathrm{IgD}^{+}, \mathrm{CD}^{-} 0^{-} \mathrm{CD}^{+}$, and $\mathrm{CD}^{-} 0^{-} \mathrm{CD} 38^{-}$populations than those of the bone marrow. The peripheral blood ROR1-expressing $\mathrm{B}$ cells had the relatively highest frequency of $\mathrm{CD} 27^{-} \mathrm{IgD}{ }^{+}, \mathrm{CD} 10^{-} \mathrm{CD}^{+}$, and $\mathrm{CD} 10^{-} \mathrm{CD} 38^{-}$population compared to those of the spleen and bone marrow, but again the majority were mainly $\mathrm{CD}_{2} 7^{-} \mathrm{IgD}{ }^{-}$and $\mathrm{CD} 10^{+} \mathrm{CD} 38^{+}$ cells. Although we observed a high percentage of $\mathrm{CD}^{+} \mathrm{B}$ cells in huNSG mice, these cells rarely expressed ROR1 (data not shown). These results indicated that ROR1-expressing B cells in huNSG mice were mostly immature human B cells. We next tested if the ROR $1^{+} \mathrm{B}$ cells can proliferate better than the ROR1 $1^{-} \mathrm{B}$ cells. It has been shown that the combination of potent $\mathrm{B}$ cell stimulators, $\mathrm{PWM}, \mathrm{CpG}$, and SAC, could lead to B cell activation and proliferation $[17,18]$. We then stimulated the splenocytes of the huNSG mice with PWM, CpG, and SAC for 4 days and measured the proliferation by fluorescence dye dilution. The unstimulated $\mathrm{ROR} 1^{+} \mathrm{CD} 19^{+} \mathrm{B}$ cells had a slightly higher percentage of proliferating cells than the unstimulated $\mathrm{ROR} 1^{-} \mathrm{CD} 19^{+}$ B cells (18.3\% versus $11.7 \%)$, while the stimulated cells had a comparable high proliferation (over 85\%).
3.4. Engraftment of TCL-1-Transduced Human CD34 ${ }^{+}$Cells in NSG Mice Could Induce ROR1-Expressing Tumors. The first transgenic mouse model of CLL was generated by overexpressing the TCL-1 gene under the control of the immunoglobulin heavy chain variable region promoter and immunoglobulin heavy chain enhancer [19]. We therefore introduced the human TCL-1 gene to human $\mathrm{CD} 34^{+}$hematopoietic progenitor cells by lentiviral transduction. A viral $2 \mathrm{~A}$ sequence was used for the simultaneous overexpression of both the TCL-1 gene and enhanced green fluorescent protein (GFP), so the TCL-1 transduction efficiency could be monitored by GFP expression. We achieved a transduction efficiency of around 40\% (Figure 5(a)), and the expression of TCL-1 in the CD34 $4^{+}$cells was confirmed by Western blotting (Figure 5(b)). The transduced cells were used to engraft newborn NSG mice. After 3 months of engraftment, most of the mice had achieved a comparable frequency of human $\mathrm{CD} 45^{+}$cells in total leukocytes, with engraftment of $\mathrm{CD} 19^{+} \mathrm{B}$ cells, $\mathrm{CD}^{+} \mathrm{T}$ cells, and $\mathrm{NKp} 46^{+}$ NK cells. Four out of the five reconstituted mice had GFPpositive cells in the periphery, with the majority being $\mathrm{CD}^{+} \mathrm{T}$ cells (Figure 5(c)). Two mice had a relatively higher proportion of GFP-positive B cells. However, the frequency of $\mathrm{ROR}^{+} \mathrm{B}$ cells in these mice was similar to that in the huNSG mice reconstituted using untransduced $\mathrm{CD} 34^{+}$cells (Figures 2(b) and 5(c)). Interestingly, the mouse with the highest engrafted $\mathrm{GFP}^{+}$cells in the periphery developed a tumor-like lump after 6 months of reconstitution. This has never been observed in other cohorts of huNSG mice engrafted with unmanipulated $\mathrm{CD} 34^{+}$cells. Single suspension cells isolated from this lump had more than $90 \%$ $\mathrm{CD} 45^{+}$human cells, without GFP expression. A high proportion of the $\mathrm{CD}_{4} 5^{+}$cells was ROR1-expressing $\mathrm{CD} 19^{+} \mathrm{B}$ cells, and more than $50 \%$ of the ROR $1^{+} \mathrm{CD} 19^{+}$cells coexpressed CD5 and CD23 (Figure 5(d)).

\section{Discussion}

In line with other findings $[15,20]$, our huNSG mice were reconstituted with all major subsets of immune cells after neonatal hematopoietic progenitor cell transplantation. huNSG mice have a high frequency of $\mathrm{CD} 19^{+} \mathrm{B}$ cells in the periphery, and over $50 \%$ of these $\mathrm{B}$ cells express the CD5 antigen [21] (data not shown). CLL patients also have a high frequency of $\mathrm{CD}^{+} \mathrm{B}$ cells in the periphery [22], and most of the CLL cases are positive for ROR1 surface expression [23]. Our data show that a high percentage of B cells in huNSG mice expresses ROR1, especially in the bone marrow and spleen.

HuNSG mice can also be generated using $\mathrm{CD} 34^{+}$hematopoietic progenitor cells isolated from umbilical cord blood or from GM-CSF-mobilized peripheral blood cells [24, 25], other than the human fetal liver. A number of studies have documented incomplete B cell development in different humanized mouse models, including the BLT mice $[21,26,27]$. If the ROR $1^{+}$immature $\mathrm{B}$ cells are indeed the by-product of incomplete B cell development in huNSG mice, it is likely that these cells are also present in other humanized mouse models. In contrast, with recent advances 


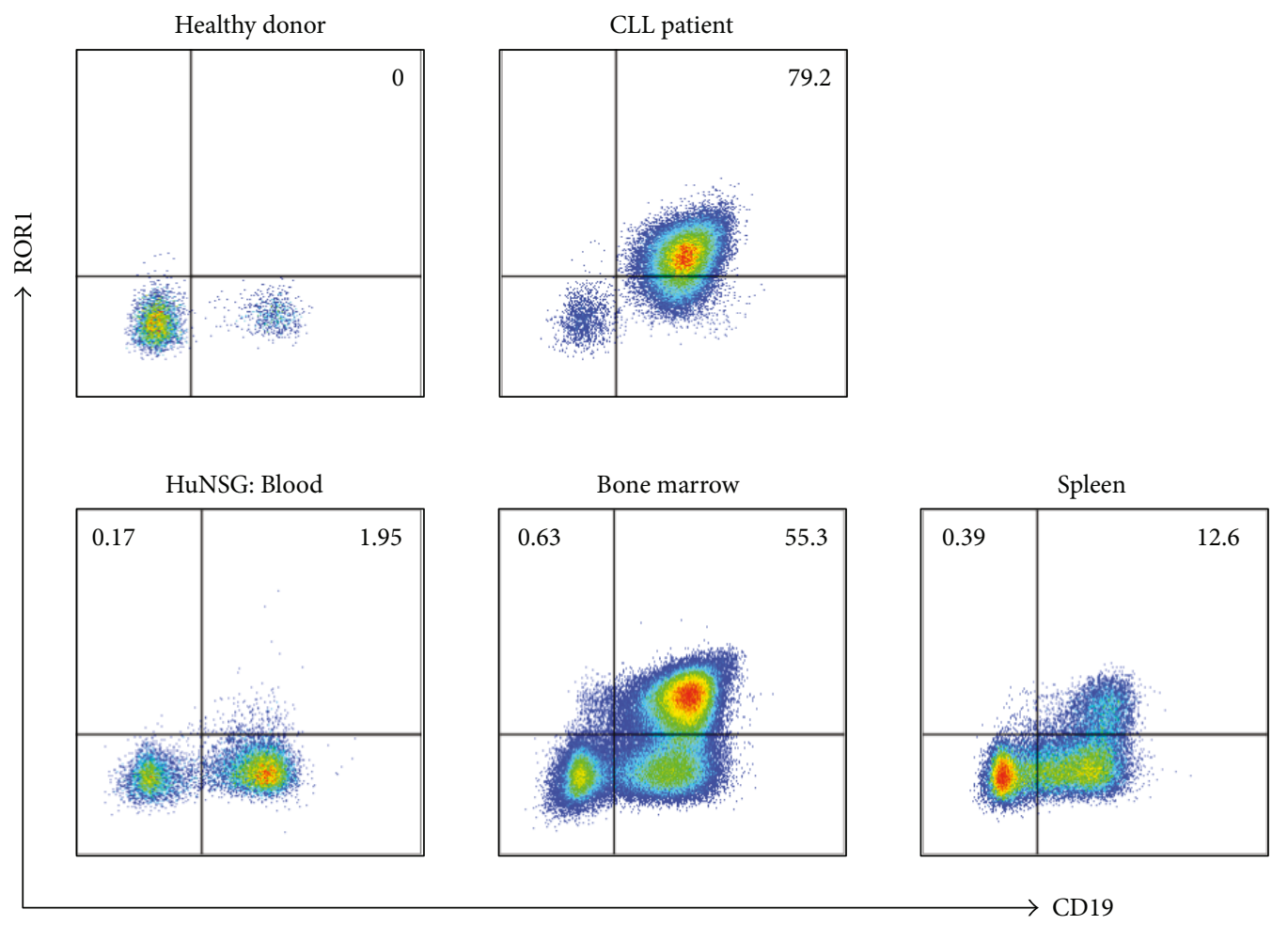

(a)

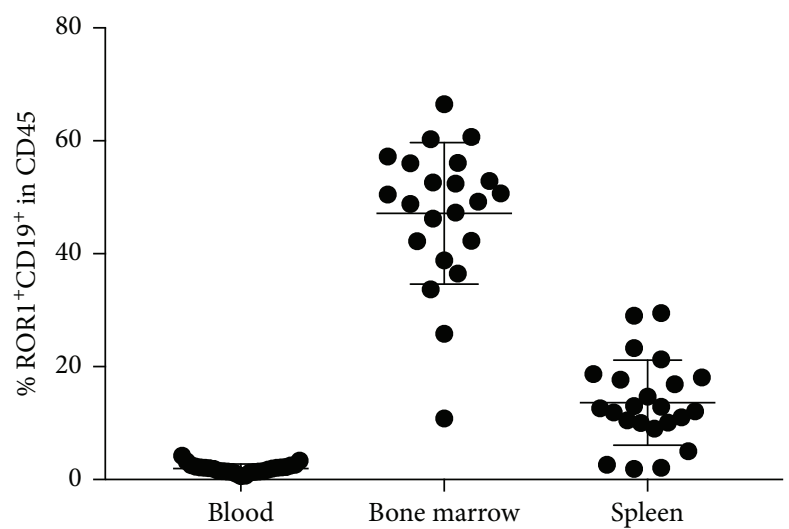

(b)

Figure 2: ROR1 expression on B cells in huNSG mice. (a) Flow cytometry staining of CD19 ${ }^{+}$and ROR1 $1^{+}$cells in PBMCs isolated from a healthy donor and a CLL patient (upper panel) and cells isolated from the blood, bone marrow, and spleen (lower panel) of huNSG mice. Samples were pregated as live cells, singlet cells positive for human CD45. The numbers indicate the frequency of CD $19^{+}$and ROR $1^{+}$cells within human $\mathrm{CD} 45^{+}$cells. (b) Composite data from 3 independent experiments are shown. Each data point represents one individually analyzed mouse. Horizontal lines represent the mean and SD.

in using new mouse strain to generate better humanized mice with improved human B cell compartment [28, 29], we would expect to see less $\mathrm{ROR}^{+}$immature $\mathrm{B}$ cells in these new models.

It has been reported that human $\mathrm{T}$ cells were required for $B$ cell maturation in a humanized mouse model [16]. This might explain the significant inverse correlation of $\mathrm{ROR}^{+}$ $B$ cells with T cells in our model. A higher number of reconstituted human $\mathrm{T}$ cells could aid B cell maturity and lead to a decrease in immature B cells. Since ROR1-expressing B cells were mostly immature $B$ cells, the numbers decreased with increasing T cells. In the case of NK cells, we did not observe a significant inverse correlation with $\mathrm{ROR} 1^{+} \mathrm{B}$ cells, suggesting that NK cells did not play a role in $\mathrm{B}$ cell maturation in huNSG mice.

ROR1 is a novel target for cancer immunotherapy as it is overexpressed in a number of malignancies without significant expression in normal adult tissues [30]. Different approaches including CAR T cells [10], monoclonal antibodies [9, 31] , and bi-specific T cell engagers (BiTEs) [32] targeting ROR1 have been developed to treat tumors. All these immunotherapies require the help of other immune cells to 
R1

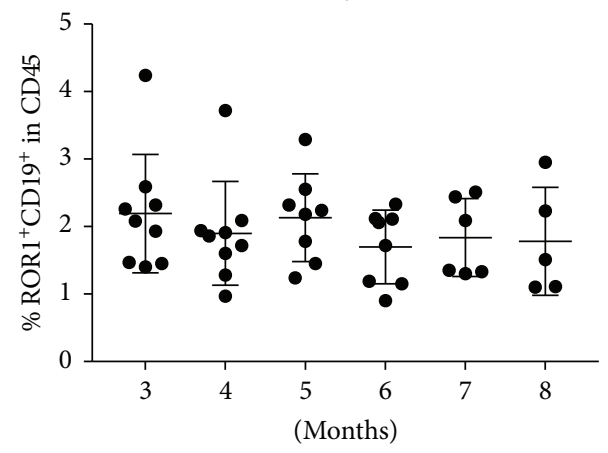

R3

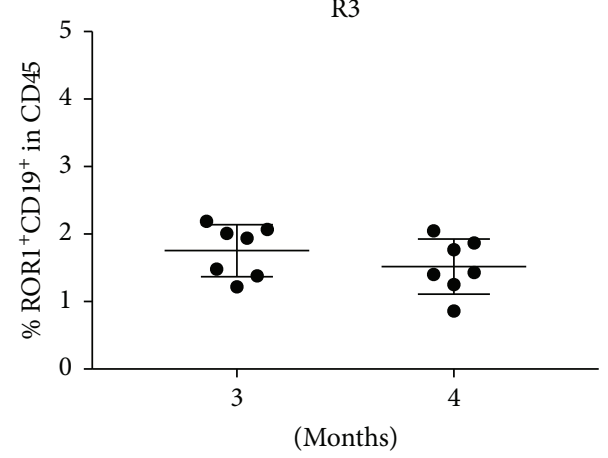

(a)

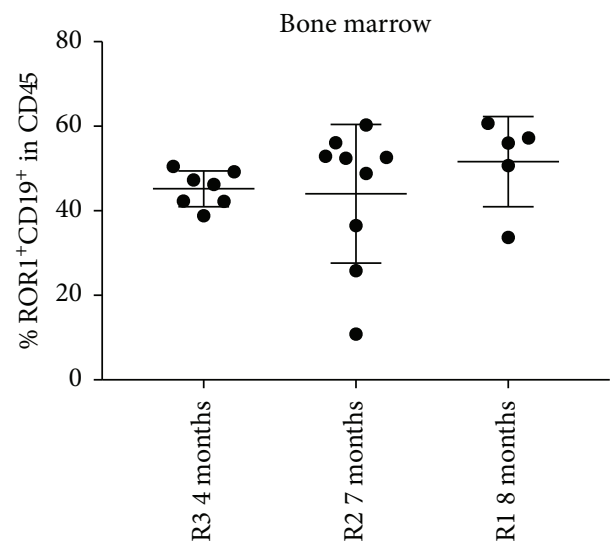

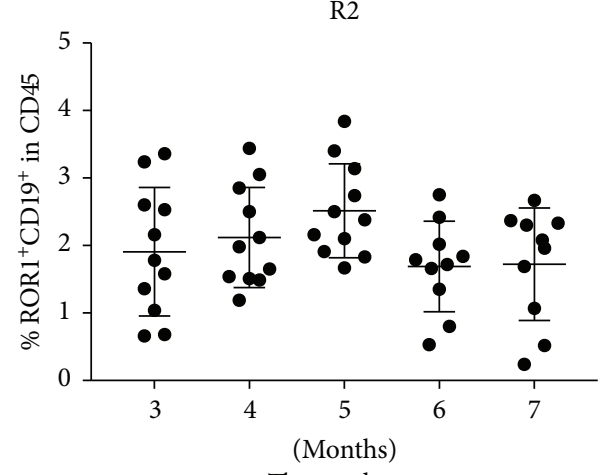

Three cohorts
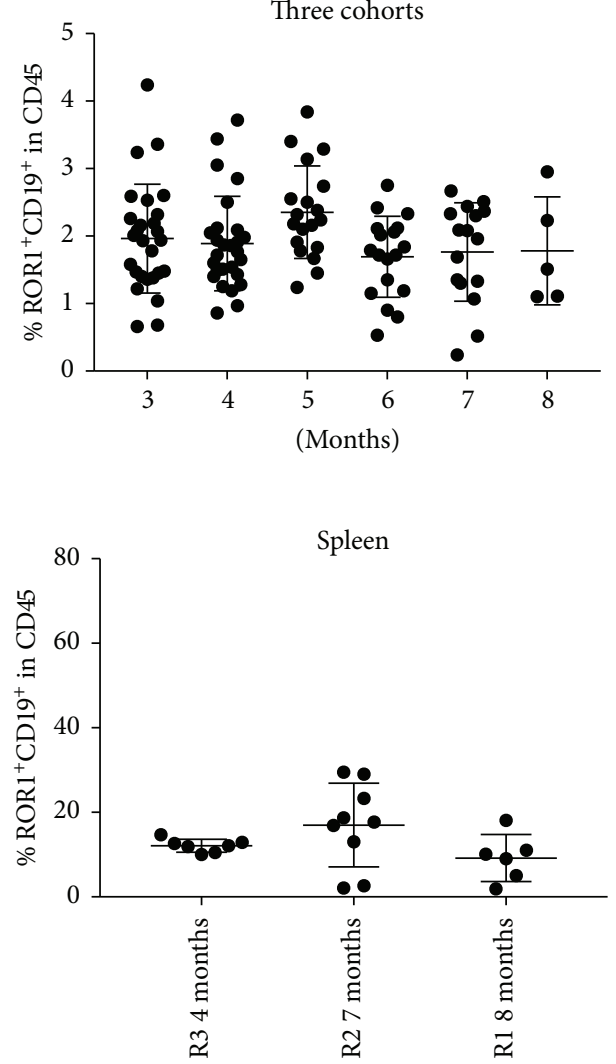

(b)
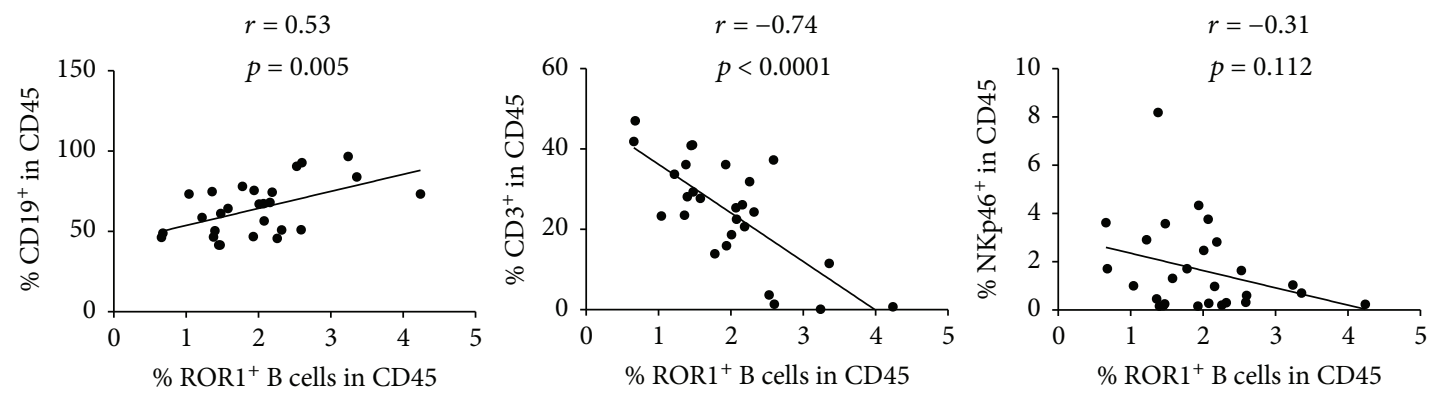

(c)

FIgURE 3: Frequency of ROR1-expressing B cells maintained in huNSG mice. (a) The frequency of ROR ${ }^{+}$B cells within human CD45 ${ }^{+}$cells in the blood of huNSG mice was analyzed in different time points after reconstitution. Data from 3 different cohorts of huNSG mice are shown individually (R1, R2, and R3) and as composite data (three cohorts). (b) Frequency of ROR $1^{+}$B cells within human CD45 cells in the bone marrow (left) and spleen (right) of huNSG mice at different time points. Horizontal lines represent mean and SD. (c) Correlation of the frequencies of ROR $1^{+} \mathrm{CD} 19^{+}$cells with the frequencies of human $\mathrm{CD} 19^{+} \mathrm{B}$ cells, $\mathrm{CD} 3^{+} \mathrm{T}$ cells, and NKp46 ${ }^{+} \mathrm{NK}$ cells in huNSG mice. The Pearson coefficient $r$ is shown, a two-tailed statistical analysis was performed, and the $p$ value is shown. 
Bone marrow
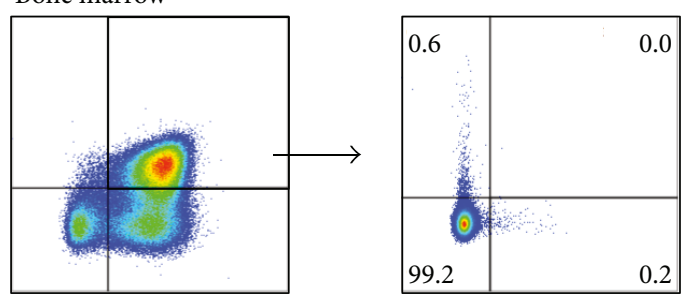

Spleen
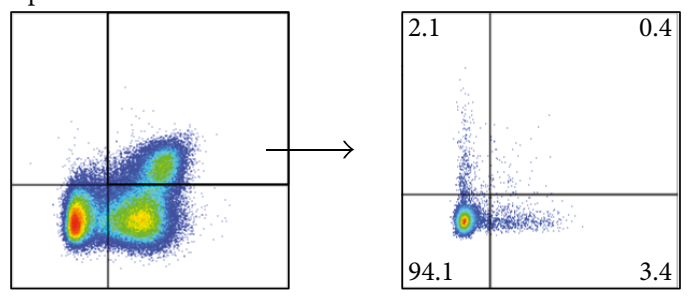
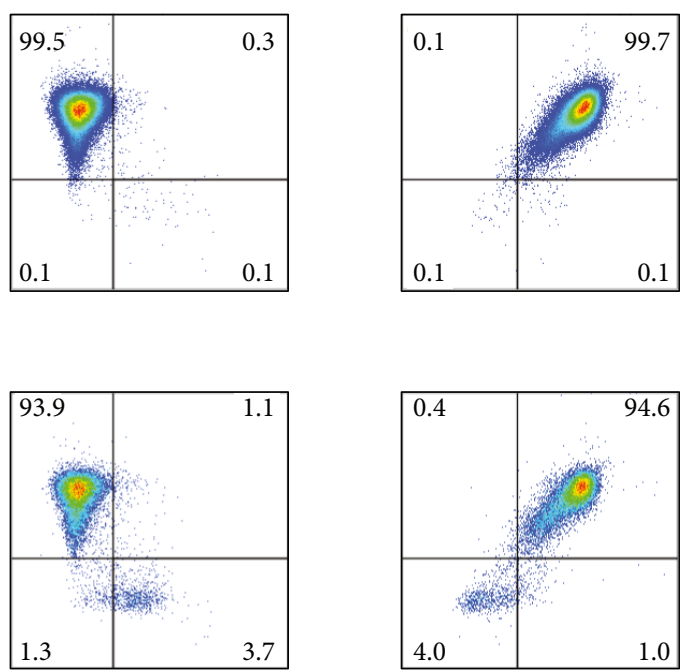

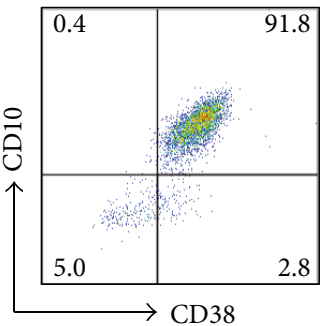

(a)

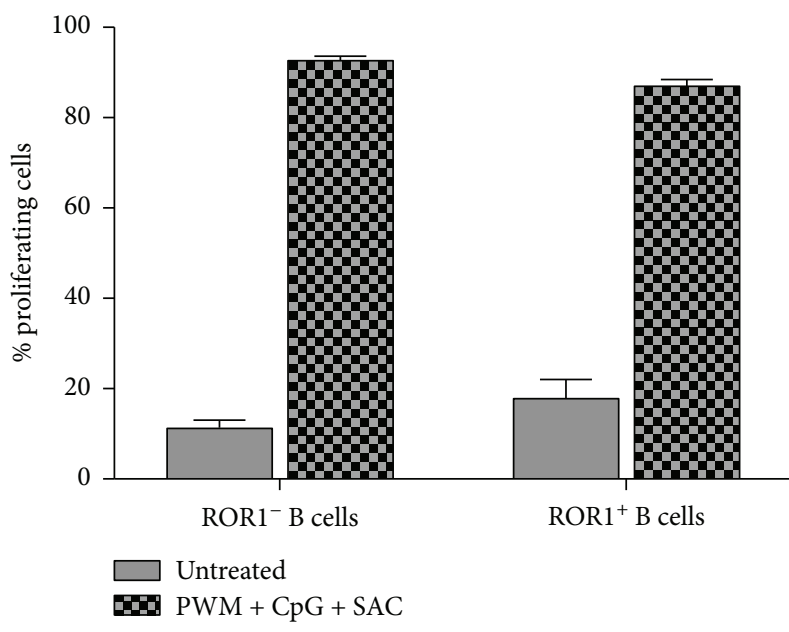

(b)

FIGURE 4: ROR1-expressing B cells were mostly immature B cells. (a) Flow cytometry staining of cells from the bone marrow, spleen, and blood of huNSG mice. Cells from the left panel were pregated as live cells, singlet cells positive for human CD45. ROR $1^{+}$CD19 $9^{+}$cells were further analyzed for their expression of CD27, IgD, CD10, CD5, and CD38. Representative data from 3 different cohorts of huNSG mice are shown. (b) Splenocytes from huNSG mice were stained with CellTrace Violet and stimulated with PWM, CpG, and SAC or left unstimulated. After 4 days, cells were harvested and stained with CD19 and ROR1 antibodies, and percentage of proliferation of live gated CD19 B cells was measured by dye dilution by flow cytometry. The $\%$ of proliferating ROR $1^{+}$and ROR $1^{-}$B cells is shown. The graph depicts the results from 2 experiments.

function. ROR1-specific CAR has to be engineered in T cells to recognize and kill ROR $1^{+}$target cells. Also, endogenous $\mathrm{T}$ cells have to be activated by BiTEs to act on ROR1 $1^{+}$tumor cells. Additionally, ROR1-specific monoclonal antibody therapy may require NK cells to mediate cytotoxicity. Therefore, an in vivo model consisting of human $\mathrm{ROR}^{+}$target cells and autologous immune cells would be a valuable tool to evaluate these therapies. Our data indicate that huNSG 


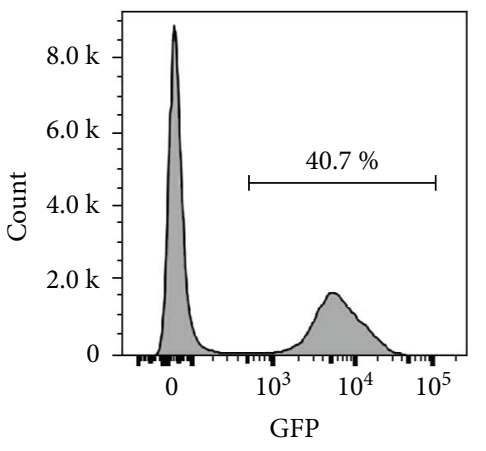

(a)

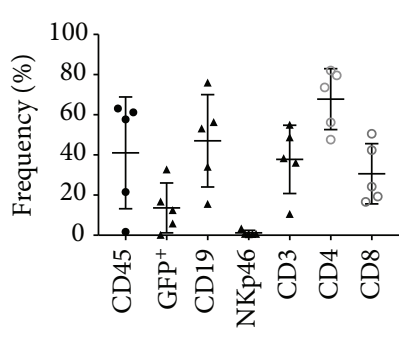

- Freq. of leukocytes

- Freq. of CD45

- Freq. of CD3

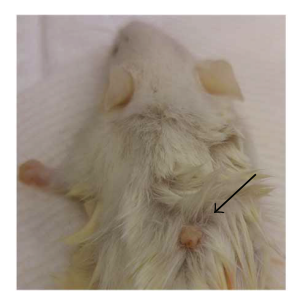

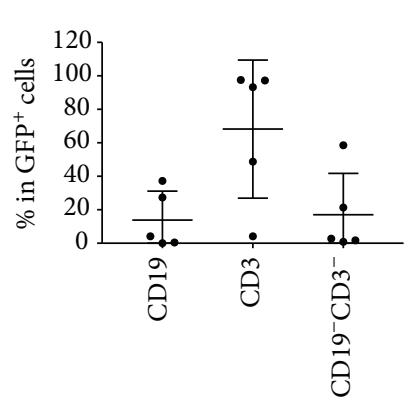

(c)

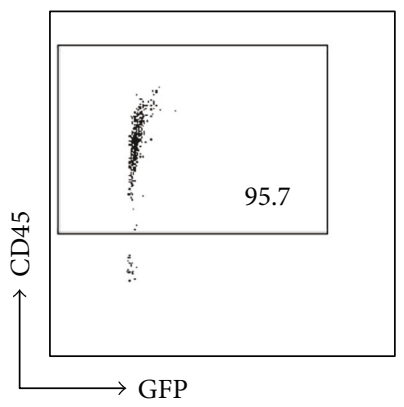

(b)
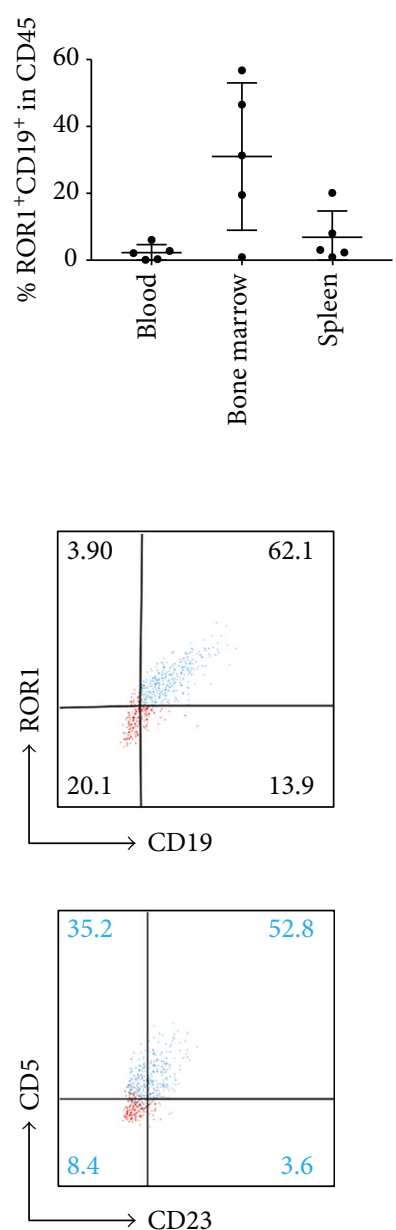

(d)

Figure 5: Engraftment of TCL-1-transduced human CD34 ${ }^{+}$cells in NSG mice could induce ROR1-expressing tumors. (a) Human CD34 ${ }^{+}$ cells isolated from fetal liver tissues were transduced with lentivirus expressing TCL-1 GFP. Frequency of GFP-positive CD34 ${ }^{+}$cells after 4 days of lentiviral transduction is shown. (b) CD34 ${ }^{+}$cells transduced with lentivirus expressing TCL-1 (TCL-1 transduced) and control lentivirus (Ctr transduced) or untransduced were harvested and lysed 4 days after infection. Protein lysates were separated by gel electrophoresis, transferred to PVDF membranes by Western blotting, and probed with TCL-1-specific antibody. The blot was also probed for GAPDH as a loading control. (c) Peripheral blood from NSG mice transplanted with lentiviral-transduced CD34 ${ }^{+}$cells was analyzed 3 months after reconstitution. The frequencies of different immune cell compartments are indicated. Frequencies of human CD45 ${ }^{+}$cells within the leukocyte gate, frequencies of $\mathrm{GFP}^{+}$cells, $\mathrm{CD} 19^{+} \mathrm{B}$ cells, NKp $46^{+} \mathrm{NK}$ cells, and $\mathrm{CD}^{+} \mathrm{T}$ cells within human $\mathrm{CD} 45^{+}$cells, and frequencies of $\mathrm{CD}^{+}$and $\mathrm{CD}^{+} \mathrm{T}$ cells within $\mathrm{CD}^{+}$cells are shown (left). The composition of the $\mathrm{GFP}^{+}$cells is shown (middle), and the frequency of $\mathrm{CD} 19^{+}$and $\mathrm{ROR}^{+}$cells within human $\mathrm{CD} 45^{+}$cells in the blood, bone marrow, and spleen of the mice is presented (right). Horizontal lines represent mean and SD. Data are from 2 different reconstitution cohorts with CD $34^{+}$cells derived from the same donor. (d) The mouse with the highest $\mathrm{GFP}^{+}$cells in the blood developed a tumor-like lump at the back as pointed by the arrow (left). Cells isolated from the tumor-like lump were analyzed by flow cytometry for the expression of human CD45 and GFP (left). The gated human $\mathrm{CD} 45^{+}$cells were further analyzed for CD19, ROR1, CD5, and CD23 expression. The ROR $1^{+} \mathrm{CD} 19^{+}$cell population is represented in blue. 
mice have a stable proportion of $\mathrm{ROR} 1^{+} \mathrm{B}$ cells in the peripheral blood, spleen, and bone marrow, together with functional autologous $\mathrm{T}$ cells and NK cells. It has been shown that huNSG T cells can be transduced and adoptively transferred back to the same host to control viral infection [33]. With this, ROR1-specific CAR T cells could be generated from huNSG mice and the efficacy can be evaluated by the removal of ROR $1^{+}$cells in the host. As we can deplete different subsets of immune cells in huNSG mice $[15,34]$, this model enables a better study of the immunotherapies.

ROR1 surface expression in B cells is detected in more than $95 \%$ of CLL cases [1]. Though we found a high percentage of huNSG B cells expressing this oncofetal antigen, these were mainly immature B cells and had a very different phenotype compared to CLL B cells. It has been reported that surface ROR1 is present at an early stage of normal B cell differentiation in human bone marrow [10]. While ROR1expressing CLL B cells are $\mathrm{CD}^{+} \mathrm{CD} 23^{+}$mature cells [35], $\mathrm{ROR}^{+} \mathrm{B}$ cells in huNSG mice are mainly $\mathrm{CD} 5^{-} \mathrm{CD} 23^{-}$ immature nonneoplastic B cells. That limits the potential use of this model to study antitumor efficacy. Moreover, this model should not be used for safety study of ROR1-directed immunotherapies because huNSG mice have a much higher frequency of ROR $1^{+}$immature B cells than humans.

We attempted to generate an in vivo CLL model by manipulating the $\mathrm{CD} 34^{+}$human hematopoietic progenitor cells. As the first transgenic mouse model of CLL was generated by overexpressing the human TCL-1 gene under the control of the immunoglobulin heavy chain variable region promoter and immunoglobulin heavy chain enhancer [19], we transduced $\mathrm{CD} 34^{+}$human hematopoietic progenitor cells with TCL-1-expressing lentivirus before injecting these cells into the neonatal mice. The reconstituted mice did not develop a CLL-like disease or other leukemic diseases. This may be due to the following reasons. First, the reconstituted GFP-positive cells, representing the engraftment of TCL-1transduced $\mathrm{CD} 34^{+}$cells, were at a relatively low level, with the highest only at $32 \%$ of $\mathrm{CD} 45^{+}$cells in one of the five mice. Second, the TCL-1 transgenic mouse model of CLL has a delayed disease development, in which CLL-like disease is usually developed at 8-12 months of age [36], whereas our mice were only at 6-7 months of age. Third, the TCL-1 gene was expressed under the $\mathrm{EF} 1 \alpha$ promoter in our model. Future study should examine if TCL-1 overexpression under the control of the immunoglobulin heavy chain variable region promoter and immunoglobulin heavy chain enhancer could lead to a CLL-like phenotype.

Although the reconstituted mice did not develop CLLlike disease, the mouse with the highest reconstituted GFP-positive cells in the blood developed a tumor-like lump. Cells isolated from the lump were human $\mathrm{CD} 45^{+}$, and the majority were ROR1-expressing B cells. More than half of these cells coexpressed CD5 and CD23, hinting that they might be neoplastic B cells. However, these cells did not express GFP, suggesting that they might not be derived from the TCL-1-transduced CD34 ${ }^{+}$cells, and how TCL-1 affected the interaction of the immune cells in huNSG mice and led to the development of this tumor-like lump remains to be determined.
In order to generate a consistent ROR $1^{+}$tumor model in huNSG mice, we have to improve the transduction efficiency of $\mathrm{CD} 34^{+}$cells and achieve a higher engraftment of TCL1-transduced $\mathrm{CD}_{34}{ }^{+}$cells [37]. The oncogenic nature of TCL-1 is well documented [38], but the addition of ROR1 overexpression in the $\mathrm{CD} 34^{+}$cells should be able to promote and drive tumorigenesis in huNSG mice [39]. Moreover, it has been shown that introducing genetic changes to $\mathrm{CD} 34^{+}$ human hematopoietic progenitor cells before injecting these cells into immunodeficient mice could generate a humanized mouse leukemic model that has recapitulating features of primary leukemia [40]. Introducing the genetic deletion of the chromosomal region 13q14, a common cytogenetic abnormality in CLL [41], could be considered.

In summary, we have shown that ROR1 protein was highly expressed in a proportion of B cells in huNSG mice; the majority of these cells were immature B cells. Transplantation of TCL-1-transduced CD $34^{+}$human hematopoietic progenitor cells in neonatal NSG mice did not increase the frequency of ROR1-expressing B cells, but the mouse with the highest engraftment of transduced cells developed a tumor-like lump consisting of a high percentage of ROR1expressing B cells. Further work would be required to induce frequent and consistent ROR $1^{+}$tumors in huNSG mice for studying immunotherapies targeting ROR1.

\section{Disclosure}

This study was presented in the poster session A (A78) at the Tumor Immunology and Immunotherapy Conference organized by the American Association for Cancer Research in October 2017.

\section{Conflicts of Interest}

The author declares no competing financial interests.

\section{Acknowledgments}

This work was supported by a fellowship from the Swiss National Science Foundation (P300P3_155374) to Carol S. Leung. The author would like to thank Mrs. Emma Knights (University College London) for her frequent checking of the general health of mice and Dr. Maria Notaridou (Autolus Limited), Dr. Xiao Qin (University College London), and Dr. Jingyi Ma (University of Oxford) for their technical help on the experiments.

\section{References}

[1] S. Baskar, K. Y. Kwong, T. Hofer et al., "Unique cell surface expression of receptor tyrosine kinase ROR1 in human B-cell chronic lymphocytic leukemia," Clinical Cancer Research, vol. 14, no. 2, pp. 396-404, 2008.

[2] V. T. Bicocca, B. H. Chang, B. K. Masouleh et al., "Crosstalk between ROR1 and the pre-B cell receptor promotes survival of $\mathrm{t}(1 ; 19)$ acute lymphoblastic leukemia," Cancer Cell, vol. 22, no. 5 , pp. $656-667,2012$. 
[3] G. Barna, R. Mihalik, B. Timár et al., "ROR1 expression is not a unique marker of CLL," Hematological Oncology, vol. 29, no. 1, pp. 17-21, 2011.

[4] A. H. Daneshmanesh, A. Porwit, M. Hojjat-Farsangi et al., "Orphan receptor tyrosine kinases ROR1 and ROR2 in hematological malignancies," Leukemia \& Lymphoma, vol. 54, no. 4, pp. 843-850, 2013.

[5] S. Zhang, L. Chen, J. Wang-Rodriguez et al., "The oncoembryonic antigen ROR1 is expressed by a variety of human cancers," The American Journal of Pathology, vol. 181, no. 6, pp. 1903-1910, 2012.

[6] A. Gentile, L. Lazzari, S. Benvenuti, L. Trusolino, and P. M. Comoglio, "Ror1 is a pseudokinase that is crucial for Metdriven tumorigenesis," Cancer Research, vol. 71, no. 8, pp. 3132-3141, 2011.

[7] M. K. Hasan, J. Yu, L. Chen et al., "Wnt5a induces ROR1 to complex with HS1 to enhance migration of chronic lymphocytic leukemia cells," Leukemia, vol. 31, no. 12, pp. 2615-2622, 2017.

[8] A. Balakrishnan, T. Goodpaster, J. Randolph-Habecker et al., "Analysis of ROR1 protein expression in human cancer and normal tissues," Clinical Cancer Research, vol. 23, no. 12, pp. 3061-3071, 2017.

[9] A. H. Daneshmanesh, M. Hojjat-Farsangi, A. S. Khan et al., "Monoclonal antibodies against ROR1 induce apoptosis of chronic lymphocytic leukemia (CLL) cells," Leukemia, vol. 26, no. 6, pp. 1348-1355, 2012.

[10] M. Hudecek, T. M. Schmitt, S. Baskar et al., "The B-cell tumorassociated antigen ROR1 can be targeted with T cells modified to express a ROR1-specific chimeric antigen receptor," Blood, vol. 116, no. 22, pp. 4532-4541, 2010.

[11] S. Meixlsperger, C. S. Leung, P. C. Ramer et al., "CD141 ${ }^{+}$dendritic cells produce prominent amounts of IFN- $\alpha$ after dsRNA recognition and can be targeted via DEC-205 in humanized mice," Blood, vol. 121, no. 25, pp. 5034-5044, 2013.

[12] L. Zitvogel, J. M. Pitt, R. Daillere, M. J. Smyth, and G. Kroemer, "Mouse models in oncoimmunology," Nature Reviews Cancer, vol. 16, no. 12, pp. 759-773, 2016.

[13] C. Leung, O. Chijioke, C. Gujer et al., "Infectious diseases in humanized mice," European Journal of Immunology, vol. 43, no. 9, pp. 2246-2254, 2013.

[14] E. Seung and A. M. Tager, "Humoral immunity in humanized mice: a work in progress," The Journal of Infectious Diseases, vol. 208, Suppl 2, pp. S155-S159, 2013.

[15] T. Strowig, C. Gurer, A. Ploss et al., "Priming of protective T cell responses against virus-induced tumors in mice with human immune system components," Journal of Experimental Medicine, vol. 206, no. 6, pp. 1423-1434, 2009.

[16] J. Lang, M. Kelly, B. M. Freed et al., "Studies of lymphocyte reconstitution in a humanized mouse model reveal a requirement of T cells for human B cell maturation," The Journal of Immunology, vol. 190, no. 5, pp. 2090-2101, 2013.

[17] I. Bekeredjian-Ding, S. Foermer, C. J. Kirschning, M. Parcina, and K. Heeg, "Poke weed mitogen requires Toll-like receptor ligands for proliferative activity in human and murine B lymphocytes," PLoS One, vol. 7, no. 1, article e29806, 2012.

[18] S. Crotty, R. D. Aubert, J. Glidewell, and R. Ahmed, "Tracking human antigen-specific memory B cells: a sensitive and generalized ELISPOT system," Journal of Immunological Methods, vol. 286, no. 1-2, pp. 111-122, 2004.
[19] R. Bichi, S. A. Shinton, E. S. Martin et al., "Human chronic lymphocytic leukemia modeled in mouse by targeted TCL1 expression," Proceedings of the National Academy of Sciences of the United States of America, vol. 99, no. 10, pp. 6955-6960, 2002.

[20] L. D. Shultz, B. L. Lyons, L. M. Burzenski et al., "Human lymphoid and myeloid cell development in NOD/LtSz-scid $I L 2 R \gamma^{\text {null }}$ mice engrafted with mobilized human hemopoietic stem cells," The Journal of Immunology, vol. 174, no. 10, pp. 6477-6489, 2005.

[21] S. Biswas, H. Chang, P. T. N. Sarkis, E. Fikrig, Q. Zhu, and W. A. Marasco, "Humoral immune responses in humanized BLT mice immunized with West Nile virus and HIV-1 envelope proteins are largely mediated via human $\mathrm{CD}^{+} \mathrm{B}$ cells," Immunology, vol. 134, no. 4, pp. 419-433, 2011.

[22] E. Matutes, K. Owusu-Ankomah, R. Morilla et al., "The immunological profile of B-cell disorders and proposal of a scoring system for the diagnosis of CLL," Leukemia, vol. 8, no. 10, pp. 1640-1645, 1994.

[23] H. E. Broome, L. Z. Rassenti, H. Y. Wang, L. M. Meyer, and T. J. Kipps, "ROR1 is expressed on hematogones (non-neoplastic human B-lymphocyte precursors) and a minority of precursor-B acute lymphoblastic leukemia," Leukemia Research, vol. 35, no. 10, pp. 1390-1394, 2011.

[24] A. Audigé, M. A. Rochat, D. Li et al., "Long-term leukocyte reconstitution in NSG mice transplanted with human cord blood hematopoietic stem and progenitor cells," BMC Immunology, vol. 18, no. 1, p. 28, 2017.

[25] J. C. Biancotti and T. Town, "Increasing hematopoietic stem cell yield to develop mice with human immune systems," BioMed Research International, vol. 2013, Article ID 740892, 11 pages, 2013.

[26] R. Vuyyuru, J. Patton, and T. Manser, "Human immune system mice: current potential and limitations for translational research on human antibody responses," Immunologic Research, vol. 51, no. 2-3, pp. 257-266, 2011.

[27] Y. Watanabe, T. Takahashi, A. Okajima et al., "The analysis of the functions of human B and T cells in humanized NOD/shi$\mathrm{scid} / \gamma \mathrm{c}^{\text {null }}$ (NOG) mice (hu-HSC NOG mice)," International Immunology, vol. 21, no. 7, pp. 843-858, 2009.

[28] H. Yu, C. Borsotti, J. N. Schickel et al., "A novel humanized mouse model with significant improvement of class-switched, antigen-specific antibody production," Blood, vol. 129, no. 8, pp. 959-969, 2017.

[29] S. Jangalwe, L. D. Shultz, A. Mathew, and M. A. Brehm, "Improved B cell development in humanized NOD-scid $I L 2 R \gamma^{\text {null }}$ mice transgenically expressing human stem cell factor, granulocyte-macrophage colony-stimulating factor and interleukin-3," Immunity, Inflammation and Disease, vol. 4, no. 4, pp. 427-440, 2016.

[30] M. Shabani, J. Naseri, and F. Shokri, "Receptor tyrosine kinaselike orphan receptor 1: a novel target for cancer immunotherapy," Expert Opinion on Therapeutic Targets, vol. 19, no. 7, pp. 941-955, 2015.

[31] J. Yang, S. Baskar, K. Y. Kwong, M. G. Kennedy, A. Wiestner, and C. Rader, "Therapeutic potential and challenges of targeting receptor tyrosine kinase ROR1 with monoclonal antibodies in B-cell malignancies," PLoS One, vol. 6, no. 6, article e21018, 2011.

[32] S. H. Gohil, S. R. Paredes-Moscosso, M. Harrasser et al., “An ROR1 bi-specific T-cell engager provides effective targeting 
and cytotoxicity against a range of solid tumors," OncoImmunology, vol. 6, no. 7, article e1326437, 2017.

[33] O. Antsiferova, A. Müller, P. C. Rämer et al., "Adoptive transfer of EBV specific $\mathrm{CD}^{+} \mathrm{T}$ cell clones can transiently control EBV infection in humanized mice," PLoS Pathogens, vol. 10, no. 8, article e1004333, 2014.

[34] O. Chijioke, A. Müller, R. Feederle et al., "Human natural killer cells prevent infectious mononucleosis features by targeting lytic Epstein-Barr virus infection," Cell Reports, vol. 5, no. 6, pp. 1489-1498, 2013.

[35] J. Hulkkonen, L. Vilpo, M. Hurme, and J. Vilpo, "Surface antigen expression in chronic lymphocytic leukemia: clustering analysis, interrelationships and effects of chromosomal abnormalities," Leukemia, vol. 16, no. 2, pp. 178-185, 2002.

[36] T. Enzler, A. P. Kater, W. Zhang et al., "Chronic lymphocytic leukemia of $\mathrm{E} \mu$-TCL1 transgenic mice undergoes rapid cell turnover that can be offset by extrinsic CD257 to accelerate disease progression," Blood, vol. 114, no. 20, pp. 4469-4476, 2009.

[37] N. Uchida, M. M. Hsieh, J. Hayakawa, C. Madison, K. N. Washington, and J. F. Tisdale, "Optimal conditions for lentiviral transduction of engrafting human $\mathrm{CD} 34^{+}$cells," Gene Therapy, vol. 18, no. 11, pp. 1078-1086, 2011.

[38] Y. Pekarsky, A. Drusco, P. Kumchala, C. M. Croce, and N. Zanesi, "The long journey of TCL1 transgenic mice: lessons learned in the last 15 years," Gene Expression, vol. 16, no. 3, pp. 129-135, 2015.

[39] G. F. Widhopf, B. Cui, E. M. Ghia et al., "ROR1 can interact with TCL1 and enhance leukemogenesis in E $\mu$-TCL1 transgenic mice," Proceedings of the National Academy of Sciences of the United States of America, vol. 111, no. 2, pp. 793-798, 2014.

[40] H. Qin, S. Malek, J. K. Cowell, and M. Ren, "Transformation of human CD34+ hematopoietic progenitor cells with DEKNUP214 induces AML in an immunocompromised mouse model," Oncogene, vol. 35, no. 43, pp. 5686-5691, 2016.

[41] U. Klein, M. Lia, M. Crespo et al., "The DLEU2/miR-15a/16-1 cluster controls B cell proliferation and its deletion leads to chronic lymphocytic leukemia," Cancer Cell, vol. 17, no. 1, pp. 28-40, 2010. 


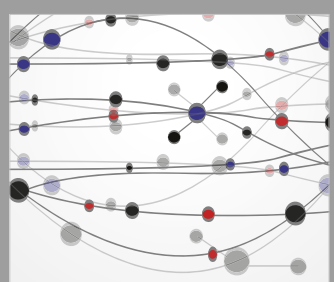

The Scientific World Journal
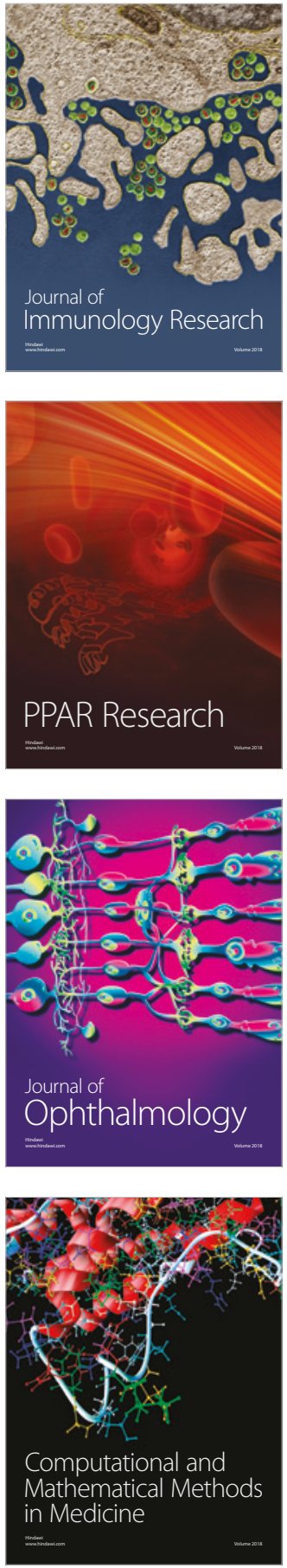

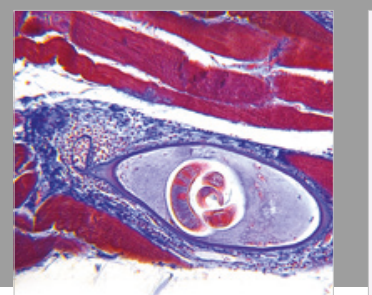

Gastroenterology Research and Practice

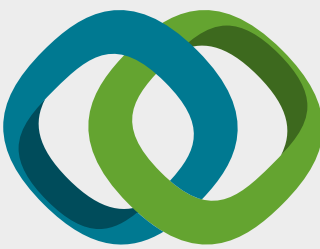

\section{Hindawi}

Submit your manuscripts at

www.hindawi.com
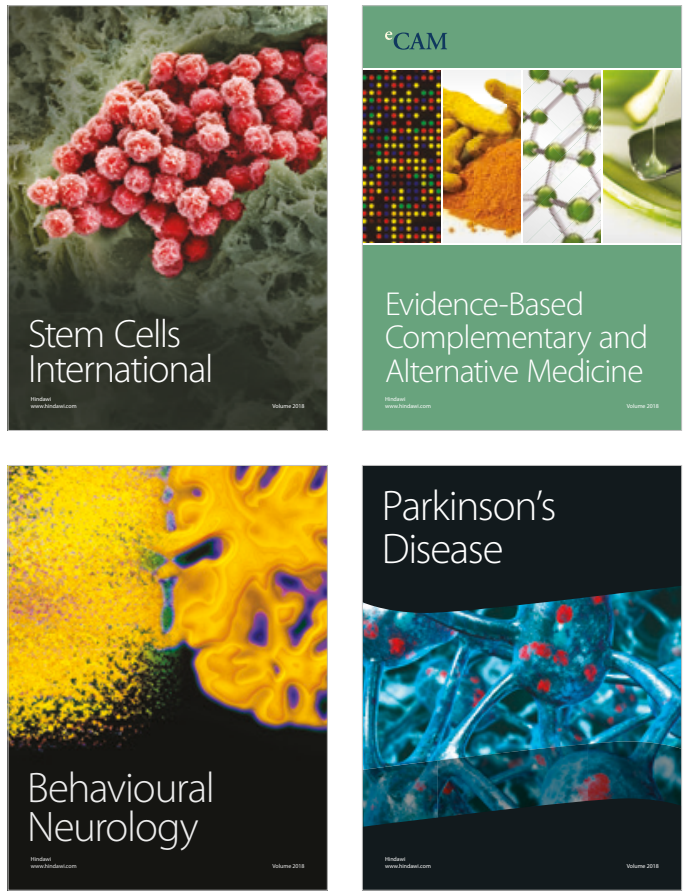

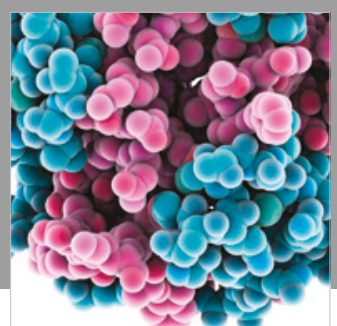

ournal of

Diabetes Research

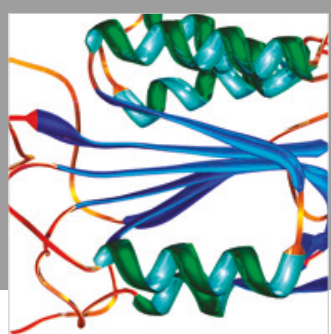

Disease Markers
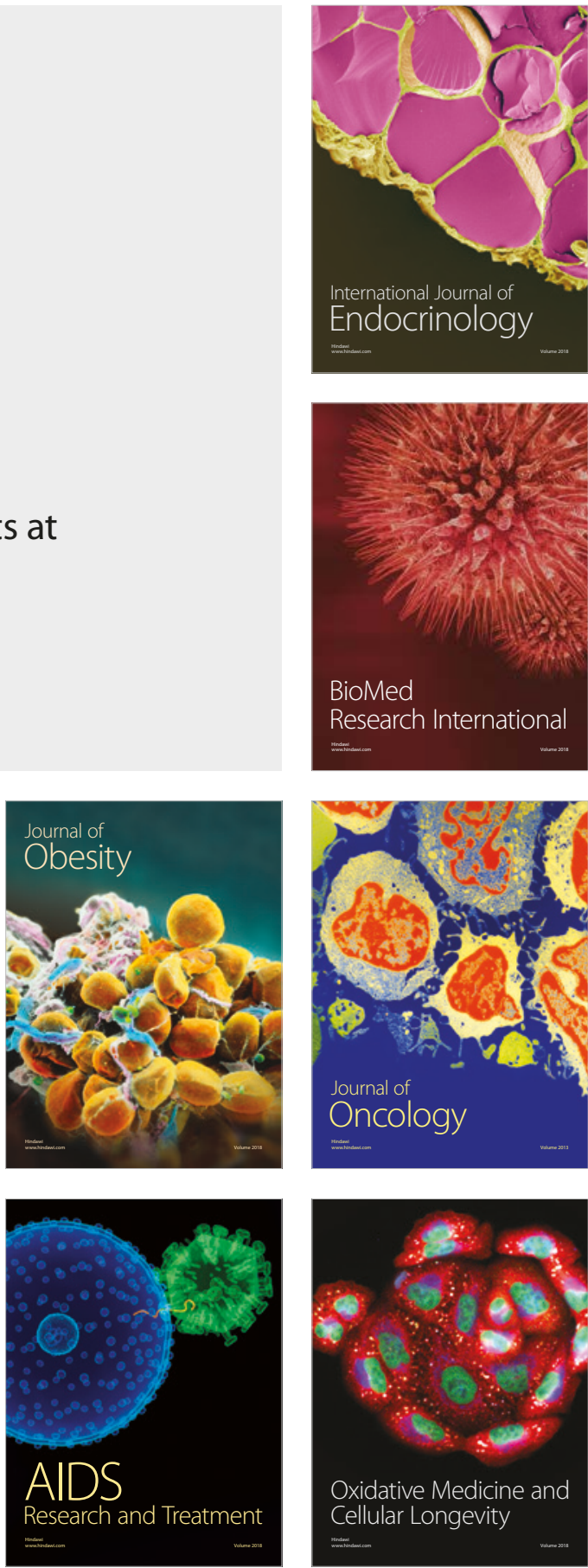